\title{
The strange physics of low frequency mirror mode turbulence in the high temperature plasma of the magnetosheath
}

\author{
R. A. Treumann ${ }^{1,2}$, C. H. Jaroschek ${ }^{3,4,1}$, O. D. Constantinescu ${ }^{5}$, R. Nakamura ${ }^{6}$, O. A. Pokhotelov ${ }^{7}$, and \\ E. Georgescu ${ }^{1}$ \\ ${ }^{1}$ Max-Planck-Institute for extraterrestrial Physics, Garching, Germany \\ ${ }^{2}$ Dept. Phys. Astron., Dartmouth College, Hanover, NH 03755, USA \\ ${ }^{3}$ Universitätssternwarte, Ludwig-Maximilians-Universität, Munich, Germany \\ ${ }^{4}$ International Max-Planck-Research-School on Astrophysics, MPG and LMU Munich, Germany \\ ${ }^{5}$ Institut für Geophysik und extraterrestrische Physik, Technische Universität, Braunschweig, Germany \\ ${ }^{6}$ Space Research Institute, Austrian Academy of Sciences, Graz, Austria \\ ${ }^{7}$ Institute of Physics of the Earth, Russian Academy of Sciences, 123810 Moscow, Russia
}

Received: 19 August 2004 - Accepted: 9 December 2004 - Published: 13 December 2004

Part of Special Issue "Advances in space environment turbulence"

\begin{abstract}
Mirror mode turbulence is the lowest frequency perpendicular magnetic excitation in magnetized plasma proposed already about half a century ago by Rudakov and Sagdeev (1958) and Chandrasekhar et al. (1958) from fluid theory. Its experimental verification required a relatively long time. It was early recognized that mirror modes for being excited require a transverse pressure (or temperature) anisotropy. In principle mirror modes are some version of slow mode waves. Fluid theory, however, does not give a correct physical picture of the mirror mode. The linear infinitesimally small amplitude physics is described correctly only by including the full kinetic theory and is modified by existing spatial gradients of the plasma parameters which attribute a small finite frequency to the mode. In addition, the mode is propagating only very slowly in plasma such that convective transport is the main cause of flow in it. As the lowest frequency mode it can be expected that mirror modes serve as one of the dominant energy inputs into plasma. This is however true only when the mode grows to large amplitude leaving the linear stage. At such low frequencies, on the other hand, quasilinear theory does not apply as a valid saturation mechanism. Probably the dominant processes are related to the generation of gradients in the plasma which serve as the cause of drift modes thus transferring energy to shorter wavelength propagating waves of higher nonzero frequency. This kind of theory has not yet been developed as it has not yet been understood why mirror modes in spite of their slow growth rate usually are of very large amplitudes indeed of the order of $\left|B / B_{0}\right|^{2} \sim \mathrm{O}(1)$. It is thus highly
\end{abstract}

reasonable to assume that mirror modes are instrumental for the development of stationary turbulence in high temperature plasma. Moreover, since the magnetic field in mirror turbulence forms extended though slightly oblique magnetic bottles, low parallel energy particles can be trapped in mirror modes and redistribute energy (cf. for instance, Chisham et al., 1998). Such trapped electrons excite banded whistler wave emission known under the name of lion roars and indicating that the mirror modes contain a trapped particle component while leading to the splitting of particle distributions (see Baumjohann et al., 1999) into trapped and passing particles. The most amazing fact about mirror modes is, however, that they evolve in the practically fully collisionless regime of high temperature plasma where it is on thermodynamic reasons entirely impossible to expel any magnetic field from the plasma. The fact that magnetic fields are indeed locally extracted makes mirror modes similar to "superconducting" structures in matter as known only at extremely low temperatures. Of course, microscopic quantum effects do not play a role in mirror modes. However, it seems that all mirror structures have typical scales of the order of the ion inertial length which implies that mirrors evolve in a regime where the transverse ion and electron motions decouple. In this case the Hall kinetics comes into play. We estimate that in the marginally stationary nonlinear state of the evolution of mirror modes the modes become stretched along the magnetic field with $k_{\|}=0$ and that a small number the order of a few percent of the particle density is responsible only for the screening of the field from the interior of the mirror bubbles. 

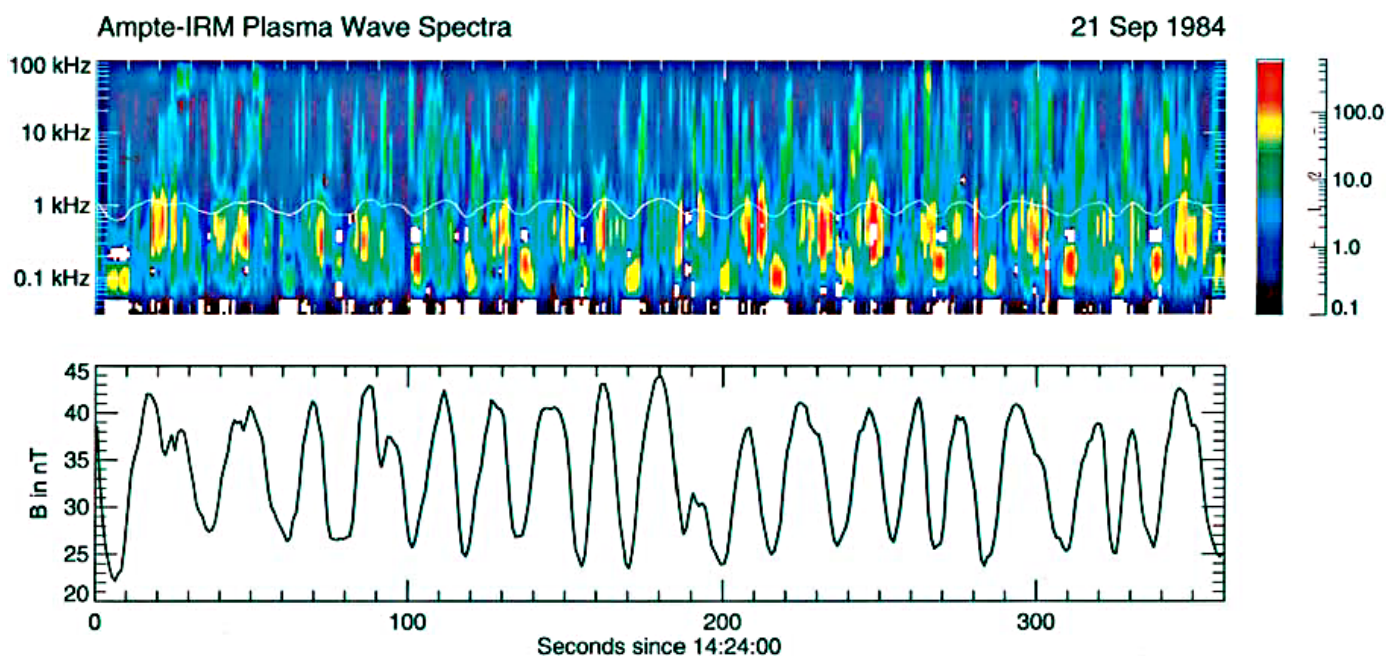

Fig. 1. Nonlinear magnetic mirror structures observed by AMPTE IRM in the magnetosheath. Lower panel: Chain of mirror mode structures in the magnetosheath outside but not far away from the magnetopause. Shown is the modulus of the magnetic field which in this case is essentially the parallel component of the field only. The transverse magnetic components are negligible. The magnetic signature is entirely compressive identifying it as the signature of mirror mode structures. The field is strictly anti-correlated with the variation of the pressure (density, not shown here). Upper panel: Wave spectrogram showing the electrostatic turbulence related to the mirror mode observations in the lower panel. The white curve gives the variation of the electron cyclotron frequency as an indication of the magnetic field modulus. The color encodes the intensity of the detected electric wave field amplitudes. Spots of intense wave fields indicate narrow band lion roar emissions in the electron whistler band signalling the presence of trapped electrons in the magnetic mirror bubbles.

\section{Introduction}

For the past quarter of a century a multitude of spacecraft observations have provided plenty of evidence for the reality of extremely low frequency magnetic structures in the solar wind (see, e.g. Winterhalter et al., 1994; Fränz et al., 2003), the magnetosheath (e.g. Kaufman et al., 1970; Tsurutani et al., 1982; Hubert et al., 1989; Lacombe et al., 1992; Hill et al., 1995; Czaykowska et al., 1998; Lucek et al., 1999, 2001), the magnetosphere (e.g. Lühr and Klöcker, 1987; Treumann et al., 1990), cometary environments (e.g. Russell et al., 1987; Glassmeier et al., 1993; Tsurutani et al., 1999) and other planetary magnetospheres (e.g. Russell et al., 1999; Huddleston et al., 1999) which all have been interpreted as so-called mirror mode structures. These structures are compressional non-oscillatory magnetic variations the typical property of which is to exhibit a pronounced local anti-correlation between the magnetic and thermal (kinetic) pressures suggesting that they are in local pressure equilibrium which at a first glance is quite reasonable assuming they represent a stationary plasma state.

This poses the non-trivial question of what kind of plasma state is represented by mirror modes. The reason is that mirror modes cause some structuring of the plasma thus generating a state of higher order than the initial state. Moreover, the plasma is ideally conducting which implies that plasma and magnetic field are frozen and cannot be separated from each other. One therefore has to find a mechanism which distorts both the frozen-in state and leads to a higher order without violating the second law, in other words, the higher order state has to be a state of reduced free energy. In the following we discuss a number of implications of these conditions on the development of mirror mode turbulence. Here we are merely posing the problem without giving a solution of the final stationary state. Any such solution has either to investigate the full dynamics of a high-temperature, ideal, turbulent plasma or to determine the thermodynamic equilibrium functions of such a plasma by minimizing the available free energy.

\section{Observational constraints}

Mirror mode structures are usually very large-amplitude depressions in the magnetic field the order of $>50 \%$ in amplitude (examples are shown in Figs. 1 and 2) sometimes even reaching $\sim 70-80 \%$ in magnetic pressure. Such structures are very far from linear state and must have formed in nonlinear interaction. Linear theory of the mirror mode applying to infinitesimally small perturbations in the magnetic field and pressure has been developed half a century ago by Rudakov and Sagdeev (1958) and Chandrasekhar et al. (1958) and in recent years has been brought to maturity (for a most recent approach see, e.g. Pokhotelov et al., 2004). However, what is observed is far beyond any linear state.

So far no reliable nonlinear theory exists that would satisfactorily describe the formation or evolution of a stationary state of the mirror mode in high temperature plasma. There have been two attempts in developing such a theory, a quasilinear approach (see, e.g. Yoon, 1992; Treumann and Baumjohann, 1997) and an approach based on modulational theory (see, e.g. Baumgärtel, 1999; Baumgärtel et al., 2003), the latter relying on the assumption that mirror modes arise 


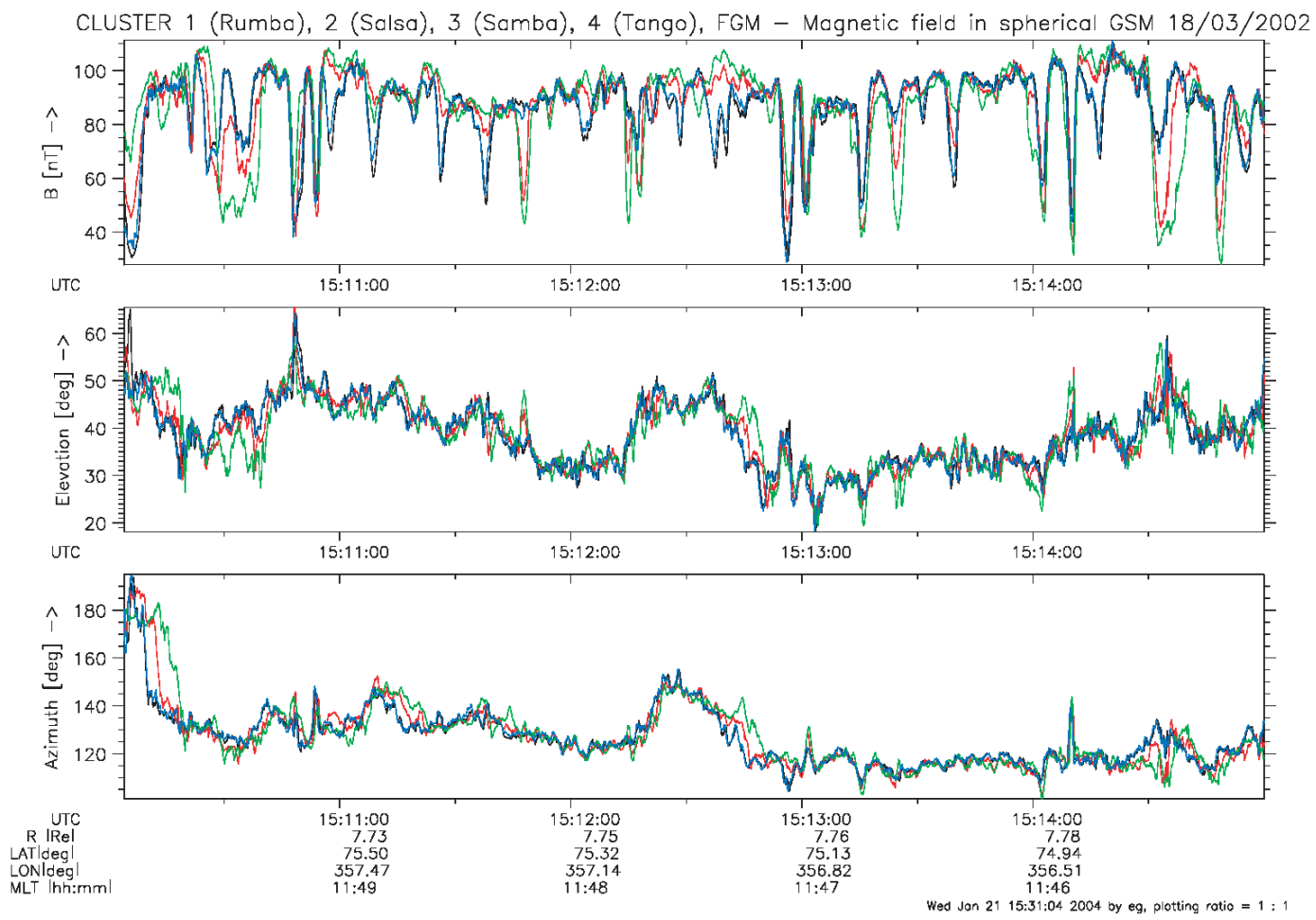

Fig. 2. A sequence of mirror mode structures similar to the AMPTE sequence as measured by the Cluster quartet in the magnetosheath (taken from Lucek et al., 2001). The deep nonlinear dropouts in the magnetic field from an in the average high magnetic field value are seen. Also seen is the weak variation of the magnetic field over the small separation distances of the Cluster spacecraft fleet. The variation is mainly in one component only as can be realized from the much more gradual (background) variations in elevation and azimuth of the magnetic fields.

basically from the nonlinear evolution of dispersive Alfvén waves. Both theories do, however, not apply.

Quasilinear theory predicts the reduction of the initial ionpressure anisotropy towards stabilization up to a minimum required for keeping the final state. Though this seems to be quite reasonable, the validity of the theory is strongly questionable because the mirror mode is a non-oscillatory wave mode of small growth rate such that averaging over the period of the wave oscillation becomes spurious. There is plenty of time for other higher frequency instabilities to develop feeded by either the plasma anisotropy or by the plasma gradients self-consistently generated by the linear mirror mode itself in the plasma. In the first case one expects ion cyclotron instabilities to arise (for a discussion see, e.g. Gary, 1992), in the second case short-wavelength highfrequency drift instabilities should evolve rapidly destroying the mirror mode.

On the other hand, theories based on solitary wave formation from dispersive Alfvénic waves referring to the derivative nonlinear Schrödinger equation (DNSE) may possibly apply to localized isolated magnetic holes observed in the solar wind ecliptic current layer (Winterhalter et al., 1994); they fail when applied to mirror mode turbulence in the currentfree magnetosheath, magnetospheres and cometary environments as there the polarization of the structures is different from single magnetic holes. First, in plasma with temper- ature (pressure) anisotropy $A=T_{\perp} / T_{\|}>1$ required for mirror modes to exist, Alfvén waves remain stable and cannot be excited unless some peculiar mechanism (like fast field aligned ion beams propagating across the plasma or local field-aligned electric potential drops serving as sources of Alfvén waves) is at work. Neither of such mechanisms exists in the magnetosheath. Second, observations show that the structures do not appear isolated but in chains of many closely located magnetic depressions separated by intense magnetic walls (see Fig. 1); third, their magnetic field polarization is entirely parallel with negligible transverse magnetic components (see for instance Fig. 3) indicating that their phase velocities are very close to being perpendicular which is in striking contrast to Alfvénic structures where the compressional component can be neglected. In addition, investigation of the particle components inside the mirror structures suggests that they contain trapped particle components which are the signature of a mirror configuration which confines the low energy component to the region of magnetic field depression. This low energy zero parallel velocity component is crucial in formation of mirror modes (as has been realized first by Southwood and Kivelson, 1993) since it is the one which is in resonance.

From this discussion it follows that the mere existence of mirror mode structures is a somewhat strange fact, in particular because in high temperature plasma a large number 

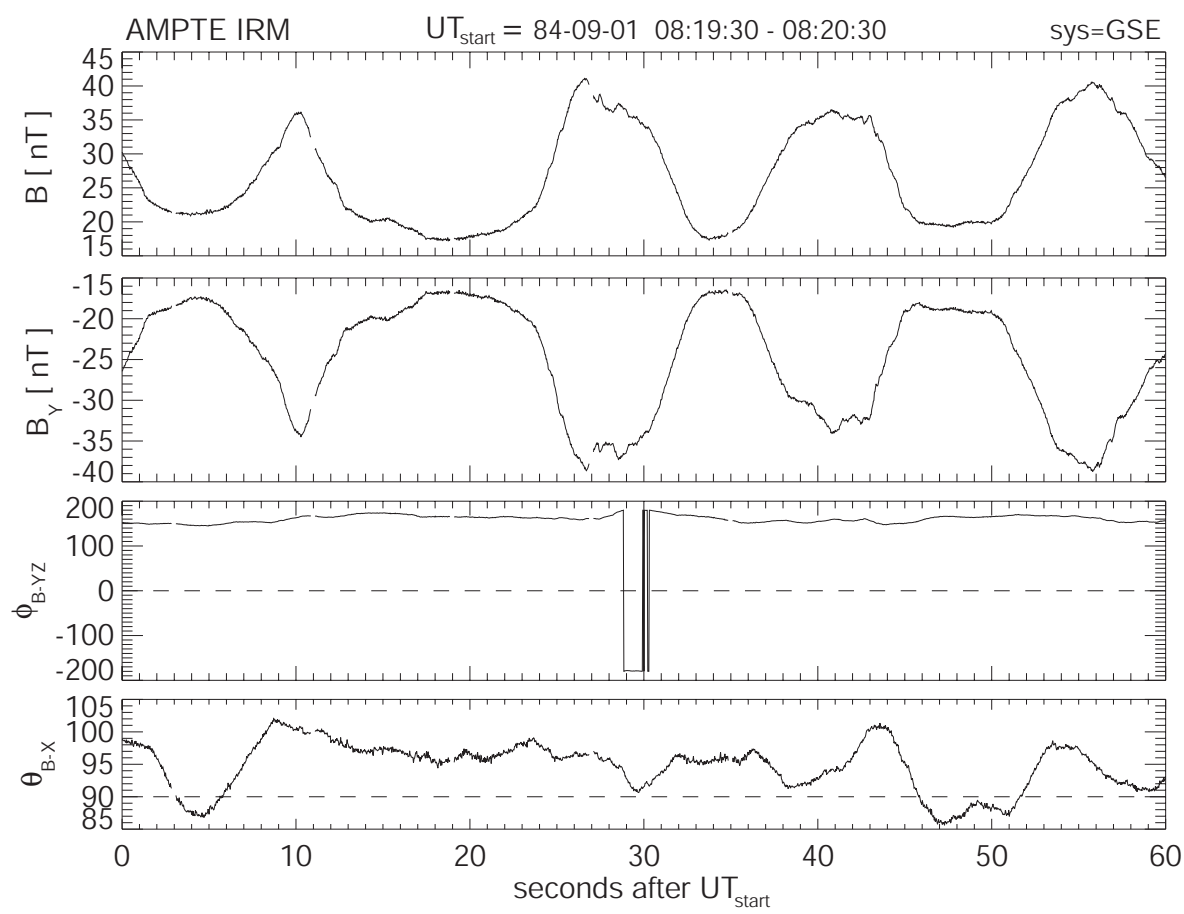

Fig. 3. One minute of AMPTE IRM mirror mode observations. Top panel: The magnitude of the magnetic field showing the highly nonsinusoidal form of the mirror bubbles with mostly broad troughs separated by steep magnetic walls. Second panel: The main magnetic component is the GSE-B ${ }_{Y}$ component. It is negative showing that the mode is completely compressive. This is also seen from the next two panels which are the GSE- $\phi$ and $\theta$ angles. $\phi$ is practically constant for the entire time interval. $\theta$ changes by $<15^{\circ}$ suggesting that the modes may have a small parallel wave vector component $k_{\|} \lesssim 0.1 k_{\perp}$ as is required by the theory of linear growth.

of turbulent modes like drift waves may arise which will fatally affect the evolution of mirror modes. On the other hand the existence of mirror bubbles suggests that they themselves represent a lowest frequency quasi-stationary turbulent mode that may evolve in a high temperature plasma leading to a distinct spatial structuring of the plasma both in the magnetic field as in the plasma pressure and densities. Mirror modes in their nonlinear state thus represent a state of minimum free energy in an anisotropic high temperature plasma with excess transverse temperature. Since structures of this kind have very low phase velocity $\omega / k \approx 0$ in the plasma frame they are subject to convection along with the plasma itself. Their frequency in the spacecraft frame is given by $\omega_{s c} \approx \mathbf{k} \cdot \mathbf{v}$ causing distinct frequency power spectra $P\left(\omega_{s c}\right)$ which map the wavelength spectrum $P(\mathbf{k})$ of the mirror modes into the frequency domain.

The spatial scale of the mirror mode can be investigated with the help of multi-spacecraft missions. Figure 4 shows two cases of (nonlinear) mirror mode observations in the magnetosheath obtained with the dual spacecraft mission AMPTE IRM-UKS in 1984. The measurements shown are three months apart. In the upper part of figure the spacecraft separation was the order of $30 \mathrm{~km}$, while in the lower part the distance between the two spacecraft was $\sim 600 \mathrm{~km}$. The striking difference between these two measurements is that at close separation distance the profiles of the mirror bubble transects are almost identical (apart from the lesser time res- olution of the UKS magnetometer) proving the stationarity and purely convective properties of the mirror mode. At the large (almost transverse) spacecraft separation, however, the similarity is lost. Thus, under the conditions prevailing in the magnetosheath the transverse spatial scale of the nonlinear mirror mode falls into the interval of a few $10<\lambda_{\perp}<600 \mathrm{~km}$. For a typical magnetosheath plasma density of $n \sim 30 \mathrm{~cm}^{-3}$, the ion inertial length is $\lambda_{i} \sim 160 \mathrm{~km}$, which is in agreement with the observations. One thus expects that the spatial scale of mirror modes is the order of $\lambda_{\perp} \sim \lambda_{i}$. This distance is also comparable with the gyroradius $r_{c i}=v_{i \perp} / \omega_{c i}$ of a magnetosheath proton. It thus suggests that the magnetosheath ions are only marginally magnetized when the mirror structures reach their nonlinear regime of stability, and ion inertia becomes important in these conditions. It is interesting enough in this respect that the linear ion-mirror instability growth rate maximizes when the wavelength of the mode becomes comparable to the ion gyroradius, though this fact must be taken with caution as the stationary state is far from linear.

\section{Summary of linear theory}

The energy source of the mirror mode is the pressure (or temperature) anisotropy of the ions. The linear theory of the mirror mode in a homogeneous anisotropic plasma has been given first by Rudakov and Sagdeev (1958) and Chandrasekhar et al. (1958) based on fluid theory. Their approach 

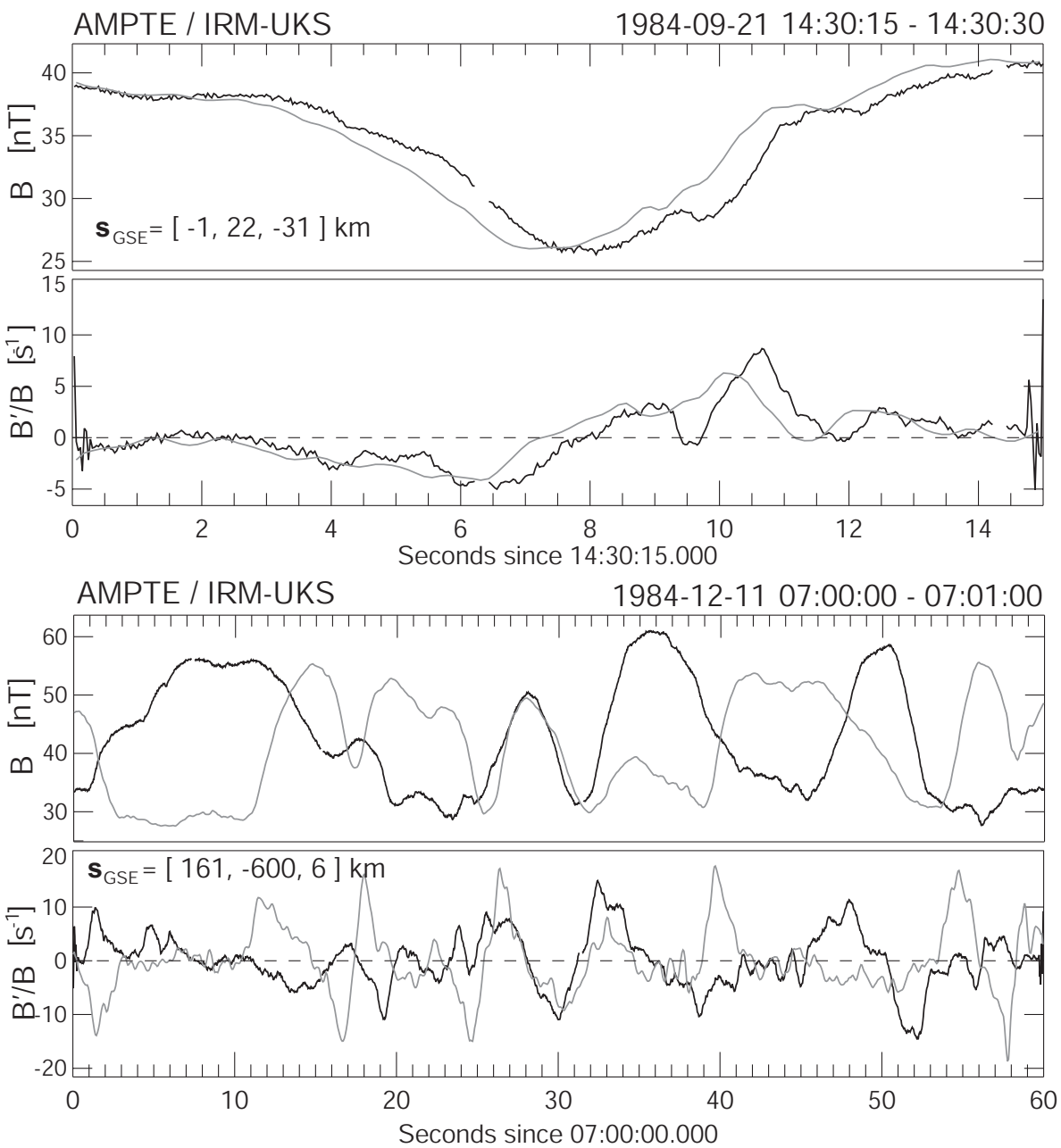

Fig. 4. Two sequences of dual AMPTE IRM-UKS spacecraft observations of magnetosheath mirror modes. The upper part of the figure is taken at small separation vector $s_{\mathrm{GSE}}=(-1,22,31) \mathrm{km}$, while the lower part of the figure is taken three months later at large separation vector $s_{\mathrm{GSE}}=(161,-600,6) \mathrm{km}$. At close separation distance only $15 \mathrm{~s}$ of measurements of the magnetic field are shown. Apart from the lower time resolution of UKS the two magnetic field profiles are identical showing that the mirror structure is stationary and is convected across the two spacecraft at magnetosheath speed. At large separation distance the longer time sequence shows that there is almost no similarity between the measurements of the two spacecraft, suggesting that the typical transverse scale of mirror modes is the order of a few $100 \mathrm{~km}$.

has later been extended to kinetic theory by Tajiri (1967) and Hasegawa (1969) who included a weak plasma inhomogeneity thus obtaining a finite real frequency of the drift-mirror mode which is caused by the diamagnetic drift frequency of the particles in the large-scale plasma gradients.

The most complete theory, including inhomogeneity, finite electron temperatures, and arbitrary distribution functions has recently been given by Pokhotelov et al. (2002) and Pokhotelov et al. (2003) where the simple quasihydrodynamic criterion for instability has been generalized. In this approach even two previously unknown new branches of the mirror mode have been uncovered, a hydrodynamic and a kinetic branch which under favorable circumstances should occur in nonuniform plasmas with finite-temperature electrons and in some cases can have larger growth rates than the ordinary ion-drift mirror mode.
The extension of linear theory to include finite ion gyroradii has been given only recently (see Pokhotelov et al., 2004). The important result of this last step is that the original finding of linear theory that the mirror growth rate increases at short wavelengths comparable to the ion gyroradius has been confirmed thereby eliminating the short-wave divergence of the growth rate at $k r_{c i} \rightarrow \infty$. Indeed, for wavelengths shorter than the ion gyroradius $k r_{c i}>1$ the growth is substantially reduced due to averaging over an ion-gyro orbit. Fastest growth is thus retained near wavelengths comparable to the gyroradius though the threshold instability is increased when taking into account the kinetic effects. In fact, it becomes dependent on wavelength such that for given temperature anisotropy onset of instability is different for different wavelengths.

The infinitesimal physics of the linear state can be read 
from the most simple linear mirror mode dispersion relation which we give here for a bi-Maxwellian plasma (Pokhotelov et al., 2004) not taking into account any finite gyroradius effects:

$$
D_{\omega, \mathbf{k}}=K-\frac{k_{\|}^{2}}{k_{\perp}^{2} \beta_{\perp}}\left(1+\frac{1}{2} \beta_{\|} A\right)+\frac{i \pi^{\frac{1}{2}} \omega}{k_{\|} v_{t h \|}} \frac{T_{\perp}}{T_{\|}}=0,
$$

where $K=A-1 / \beta_{\perp}$, and $A=\left(T_{\perp} / T_{\|}\right)-1$ is the temperature anisotropy. The term $A$ results from magnetically "mirroring" particles, i.e. from the reflection of particles from regions of increasing magnetic field by a quasi-static compressive field perturbation (e.g. Southwood and Kivelson, 1993). The term $1 / \beta_{\perp}$ is the elastic resistance of the magnetic field lines; it decreases with growing $\beta_{\perp}$. The next term in the above equation contains the effect of magnetic field line bending which contains the difference $\beta_{\perp}-\beta_{\|}$from the pressure anisotropy. The last term is the only one which contains the frequency. This term is the contribution of resonant particles which in this case have zero parallel velocity; with respect to the parallel phase velocity of the wave these particles are at rest along the ambient magnetic field. Solving for the frequency yields the growth rate

$\gamma=\frac{k_{\|} v_{t h \|}}{\pi^{\frac{1}{2}}} \frac{T_{\|}}{T_{\perp}}\left[K-\frac{k_{\|}^{2}}{k_{\perp}^{2} \beta_{\perp}}\left(1+\frac{1}{2} \beta_{\|} A\right)\right]$.

This growth rate has been extensively discussed. It contains the competition between the tension generated by the field line bending and the ballooning pressure resulting from the pressure anisotropy. We note here that it requires $k_{\|} \neq 0$ and

$K>\frac{k_{\|}^{2}}{k_{\perp}^{2} \beta_{\perp}}\left(1+\frac{1}{2} \beta_{\|} A\right)$

and for given anisotropy $A, \beta_{\|}, \beta_{\perp}$ and $k_{\perp}$ maximizes for a propagation angle $\theta$ between $\mathbf{k}$ and the magnetic field given by

$$
\tan ^{2} \theta=\frac{1}{3}\left(\frac{\beta_{\perp} A-1}{1+\beta_{\|} A / 2}\right) .
$$

This yields a growth rate at maximum unstable angle

$\gamma_{\theta}=\frac{v_{t h \|}}{A+1}\left[\frac{4 \beta_{\perp}}{27 \pi} \frac{K^{3}}{\left(1+\frac{1}{2} \beta_{\|} A\right)}\right]^{\frac{1}{2}}$.

Finite gyro radius effects introduce some further modifications (Pokhotelov et al., 2004) which do not interest us here. Instead we note that the above growth rate suggests that under marginally stable conditions when the mode growth ceases due to the action of some nonlinear effects the mode will propagate strictly parallel with $k_{\| \operatorname{marg}}=0$ and $\gamma_{\text {marg }}=0$. Under such a condition we will thus have $K_{\text {marg }} \equiv 0$ and, hence,

$$
A_{\text {marg }} \beta_{\perp \text { marg }}=1,
$$

which is the original threshold condition for the linear mirror mode. One may therefore expect that under nonlinearly stable conditions the mirror mode will have a number of distinct properties:

- Its lowest free energy state will be characterized by strictly perpendicular propagation with zero field line bending;

- mirror mode bubbles will therefore consist of extended magnetic flux depressed tubes being separated from each other by magnetic walls;

- the remaining marginal pressure anisotropy will strictly satisfy the marginal stability condition;

- the typical perpendicular scale of the flux depressed tubes will roughly be the order of the thermal ion gyroradius (or ion inertial length), because the growth rate maximizes for wavelengths comparable to the gyroradius.

\section{Nonlinear treatment: a superconducting analogue}

The nature of the nonlinear evolution of the mirror mode to form highly nonlinear stationary structures of the kind which is observed in the magnetosheath is of course not easily inferred. Knowing that the condition of marginal stability should also describe the final nonlinear state and that in such a state the mirror mode should be essentially twodimensional with transverse size of the order of the ion inertial length, we can interpret the condition of marginal stability as the critical condition separating two basically different states of plasma, a normal state, when the magnetic field is large, and a special state referring to the interior of mirror structures when the magnetic field is reduced. In doing so, and noting that $\beta=2 \mu_{0} n k_{B} T / B^{2}$, with $n$ the density, we may rewrite the condition of marginal stability as a condition on the magnetic field

$B_{S}<B_{c 1} \equiv\left[2 \mu_{0} n k_{B} \frac{T_{\perp}}{T_{\|}}\left(T_{\perp}-T_{\|}\right)\right]^{\frac{1}{2}}$,

where the index $c$ denotes the critical value of the magnetic field. For magnetic fields less than this critical value the plasma will consist of a system of mirror bubbles while for larger magnetic field it will assume its normal bubble-free state. The special state $B_{s}$, when the mirror bubbles evolve will thus be a plasma state different from normal and the plasma will undergo a phase transition when changing from normal to special states, respectively.

The above condition shows that the critical magnetic field depends on the temperature in a special manner. For $T_{\|}>T_{\perp}$ the critical magnetic field becomes imaginary and no phase transition occurs. On the other hand, when $T_{\|}<T_{\perp}$ phase transitions become possible. Therefore, $T_{\perp}$ plays the role of a critical temperature for $T_{\|}$, and we can define a temperature scale $\vartheta=T_{\|}-T_{\perp}$ such that for $\vartheta>0$ the system is in normal 
state while for $\vartheta<0$ it undergoes phase transition to a state of (stationary) mirror turbulence. In this case we write

$B_{c 1}=\left[\frac{2 \mu_{0} n k_{B} T_{\perp}}{T_{\perp}+\vartheta}(-\vartheta)\right]^{\frac{1}{2}}$.

Close to marginality we have $|\vartheta| / T_{\perp} \ll 1$ such that the small correction in the denominator can be neglected and

$B_{c 1} \approx\left[2 \mu_{0} n k_{B}(-\vartheta)\right]^{\frac{1}{2}}$.

From here we find that close to critical conditions the critical magnetic field scales with temperature as

$B_{c 1} \propto(-\vartheta)^{\frac{1}{2}}$.

Such a scaling bears a certain similarity to the Meissner effect in metal superconductivity where the critical magnetic field would scale as $B_{c M} \propto-\vartheta$.

The critical field $B_{c 1}$ marks the transition from the normal to the mirror structured plasma states, respectively. There exists another critical field $B_{c 2}$ such that for smaller magnetic fields $B<B_{c 2}$ the entire plasma becomes demagnetized. This critical field is determined by the condition that the many neighboring mirror structures start overlapping. For this to happen the perpendicular scale $k_{\perp}^{-1}$ of the mirror structures must exceed their distance $d$ of separation. Little is known about $d$, however. Figures 1 and 3 suggest that $d$ is less or comparable to $k_{\perp}^{-1}$. The mere existence of chains of mirror bubbles suggests on the other hand that the typical state when they are observed is the mixed state consisting of mirror structures and magnetic walls.

Indeed, an extended region of decreased magnetic field with steep walls would hardly be recognized as a huge mirror structure in non mixed state and might not exist at all.

The interesting remaining question is what keeps the mirror structures free of magnetic field. In linear mirror mode theory the assumption is that the pressure balance between cold plasma streaming into the bubbles from regions of high field strength balances the magnetic field pressure of the separating walls. However, we have seen that this condition is broken in the final stable state when the growth rate vanishes and the bubbles stretch into infinity along the magnetic field. In this minimum free energy case the stability of the bubbles is assured in addition to pressure balance by diamagnetic screening currents flowing along the surfaces of the bubbles. The number density of particles involved into these currents is $n^{*}$, and the width of the current layer is the (ion) screening length $\lambda_{i}^{*}=c / \omega_{p i}^{*}$ based on $n^{*}$. Measuring the screening length of the magnetic field thus allows for the determination of $n^{*}$.

Guided by the London theory let us assume that, in the simplest case, the magnetic field decreases into the mirror bubble as $B(x) \propto \exp \left(-x / \lambda_{p i}^{*}\right)$ with distance $x$ from the wall and that the structures are convected across the spacecraft at (magnetosheath) flow velocity $v_{\mathrm{msh}}$. Then

$\lambda_{i}^{*}=-\frac{\mathrm{d} x}{\mathrm{~d} \ln B}=-\frac{v_{\mathrm{msh}} \mathrm{d} t}{\mathrm{~d} \ln B}$.
In terms of measured quantities this yields for the density of the "superconducting" particles

$\frac{n^{*}}{n_{0}}=\frac{c^{2}}{\omega_{p e 0}^{2}}\left|\frac{\mathrm{d} \ln B}{v_{\mathrm{msh}} \mathrm{d} t}\right|^{2}$.

Using the numbers in the upper part of Fig. 3, we find from pressure balance for the density enhancement in the mirror bubble

$\Delta n=\frac{B_{0}^{2}}{2 \mu_{0} k_{B} T}\left(1-\frac{B_{m}^{2}}{B_{0}^{2}}\right) \approx 0.9 \frac{B_{0}^{2}}{2 \mu_{0} k_{B} T} \simeq 30 \mathrm{~cm}^{-3}$,

where we used $k_{B} T \approx 30 \mathrm{eV}, B_{0}=30 \mathrm{nT}$, and for the minimum in the magnetic field $B_{m} \simeq 0.3 B_{0}$.

Figure 3 (lower panels) shows the logarithmic derivatives taken across the mirror structures. As the relevant value we take the maximum $B^{\prime} / B=-5$ of the logarithmic derivative in the descent of the magnetic field of the upper part as it corresponds to the turning point in the gradient of the magnetic field. From the above expression and the above values for $n_{0}$ we then find that

$n^{*} \approx 0.16 / v_{\mathrm{msh}}^{100} \mathrm{~cm}^{-3}$,

where $v_{\mathrm{msh}}^{100}=v_{\mathrm{msh}} / 100 \mathrm{~km} \mathrm{~s}^{-1}$. The flow speed was $v \sim 50 \mathrm{~km} \mathrm{~s}^{-1}$. From these values we find $n^{*} \approx 0.7 \mathrm{~cm}^{-3}$, or

$n^{*} / n_{0} \approx 2 \%$,

suggesting that only a fraction the order of a few per cent of the magnetosheath plasma is involved into the diamagnetic currents which (partially) screen the mirror bubbles from the magnetic field.

From these considerations we may conclude that the final state of the mirror modes is in agreement with the assumption that some of the trapped plasma in mirror bubbles partially screens the magnetic field from the interior of the bubble. The mechanism of this screening is not identical though similar to the Meissner effect in superconductivity (see, e.g. Huang, 1995, Chp. 10) though in this case only classical particles are involved and no pairing of particles exists. Initially during evolution of the mirror mode particles of low parallel energy are confined by the mirror force into the mirror bubble. When the mirror mode relaxes toward nonlinearity its parallel wave number vanishes, and the trapped particles generate a screening current flowing along the surface of the bubble. As in the Meissner effect the diamagnetic effect of these currents is responsible for the persistent evacuation of the magnetic flux. The mirror-unstable high temperature plasma therefore behaves in some of its properties analogous to a superconductor. This is not as surprising as it sounds since the plasma is anyway ideally conducting, and the only necessary property that is required to produce the analogy is the property of macroscopic diamagnetism. This is warranted by the generation of a diamagnetic current $\boldsymbol{j}=\left(\omega_{p i}^{* 2} / c^{2}\right) \boldsymbol{A}$, with $\boldsymbol{A}$ the magnetic vector potential, flowing in the transition layer between the mirror structure and the magnetic walls. 
The mirror-unstable plasma represents a type II superconductor in mixed state containing a large number of magnetic flux tubes filling the regions between the magnetic field evacuated mirror bubbles. It should in principle be possible to describe such a medium by an equation of the kind of the Ginzburg-Landau equation (see, e.g. Huang, 1995; Lifschitz and Pitaevskii, 1998, the chapters on the phenomenological theory of superconductivity). An attempt to derive such an equation is under way but is beyond the intention of the present paper. We note, however, that in type II superconductors the ratio $\kappa=\lambda_{i}^{*} / \xi>1 / \sqrt{2}$, where $\xi$ is the correlation length of the screening particles. In the absence of a microscopic theory of the final stationary state the latter is not known. Since most of the particles are involved in the screening it is reasonable to assume that the correlation length corresponds to the density gradient which for bubbles being in pressure equilibrium is the same as $\lambda_{i}^{*}$, in which case $\kappa \approx 1$ marginally satisfying the above condition. The ratio $\kappa$ (for metallic superconductors) can be expressed phenomenologically (see, e.g. Lifschitz and Pitaevskii, 1998, Ch. 5, pp. 153183 ) in terms of measurable quantities as

$\kappa=2 \sqrt{2}\left(\frac{e}{\hbar m}\right) B_{c 1}(\vartheta) \delta^{* 2}(\vartheta)$,

where $\delta^{*}$ is the London penetration depth of the magnetic field. Let us assume that dimensionally a similar expression holds for mirror modes in their nonlinearly stationary (marginally stable) state. Since $\kappa$ must be constant and independent of temperature $\vartheta$, we find that the mirror mode screening length $\delta^{*}=\lambda_{i}^{*}$ scales with temperature as

$\lambda_{i}^{*}(\vartheta) \propto(-\vartheta)^{-\frac{1}{4}}$.

There is no direct equivalent in the classical case for the above expression of $\kappa$. The constant $\hbar$ arises solely from the definition of the momentum operator $\hat{p}=i \hbar \nabla$ in the quantum treatment of the Ginzburg-Landau theory. If we tentatively replace $\hbar \rightarrow m c \lambda_{i}$ in our classical case, we can express

$\kappa=2 \sqrt{2}\left(\frac{n_{0}}{n^{*}}\right)\left(\frac{m_{i}}{m_{e}}\right)\left(\frac{v_{A}}{c}\right)$,

where $v_{A} \approx 100 \mathrm{~km} \mathrm{~s}^{-1}$ is the Alfv'en speed, and we assumed that the mass $m=m_{e}$ in the replacement of $\hbar$ is the electron mass. Inserting for the density ratio we find in this case $\kappa \approx 100 \gg 1$ suggesting that the mirror modes are definitely in the mixed state. However, when using the ion mass in the above replacement the mass ratio is one, and the value of $\kappa$ can be either larger or smaller than $1 / \sqrt{2}$ depending on the exact values of the Alfvén speed. This uncertainty is related to the incomplete knowledge of the correlation length of the "superconducting" particles. Hence, unless a more precise theory of this correlation length can be offered, the value of $\kappa$ remains undetermined and thus inconclusive. For the measurements shown in Fig. 4 (upper part), it seems that $\kappa>1$ suggesting that mirror modes are indeed located deep inside the domain of type II superconductors and thus appear in mixed state.
We mentioned earlier that the linear growth rate maximizes near $k_{\perp} r_{c i} \approx 1$, taking into account the finite gyroradius effects. This does not substantially change the above discussion. The main variation is in the marginal anisotropy which now becomes $A=1 / \beta_{\perp}^{*}$ (Pokhotelov et al., 2004) where $\beta_{\perp}^{*}=\beta_{\perp}\left[I_{0}(z)-I_{1}(z)\right] \mathrm{e}^{-z}$, with $z=k_{\perp}^{2} \lambda_{i}^{* 2} \beta_{\perp}$, and $I_{0}, I_{1}$ the modified Bessel functions of zero and first order. Since now the marginal magnetic field is contained in the argument of the Bessel functions, the expression for $B_{c 1}$ becomes more complicated. One, however, observes that for long (perpendicular) wavelengths the condition is the one given above, while for short wavelengths $k_{\perp} \rightarrow 0$ and the threshold condition increases implying a vanishing critical magnetic field which eliminates the effect. Thus the most probable perpendicular scale of the modes will still be close to $k_{\perp} \lambda_{i}^{*} \approx 1 / \sqrt{\beta_{\perp}}$. which is in approximate agreement with observation.

\section{Summary and conclusions}

The main result of this investigation is that mirror modes at their nonlinear final stationary stage tend to become long stretched magnetic field-depleted tubes with $k_{\|}=0$ distributed across the plasma volume in a mixed state of such tubes and magnetic walls separating the tubes. This state in the ideally conducting collisionless plasma of space corresponds to a type II "superconducting mixed state" similar to a Ginzburg-Landau (or Abrikosov-Gor'kov) state familiar from low-temperature solid state superconducting theory. High-temperature anisotropic collisionless plasmas thus may under the condition of Eq. (9) resemble such superconductors.

The "superconducting" property is warranted by the property of localized diamagnetism in the plasma which under normal conditions does not evolve. It also manifests itself in the obvious suppression of other local instabilities which could otherwise generate anomalous resistivity. Most of these facts are still barely understood as one would expect that the steep magnetic field and density gradients generated could cause evolution of other micro-instabilities which tend to deplete the gradients. Since mirror structures are very long-lived subject there is plenty of time for such instabilities to evolve. Because of these facts we speak of the strange physics of mirror modes, which can be illuminated only by developing a consistent statistical mechanical theory of the mirror mode equilibrium, viz. minimization of the available free energy in an anisotropic collisionless high-temperature $\beta \gtrsim 1$ plasma. Currently we are far away from such a goal which possibly can be achieved only by full-particle numerical simulation.

We have derived an expression for the critical magnetic field strength $B_{c 1}$ for onset of the corresponding (superconducting) phase transition from normal to mirror state. This critical field is a function of temperature difference $\vartheta$ between parallel and critical temperatures. As critical temperature $T_{\| c}$ the perpendicular temperature $T_{\perp}$ of the plasma has 


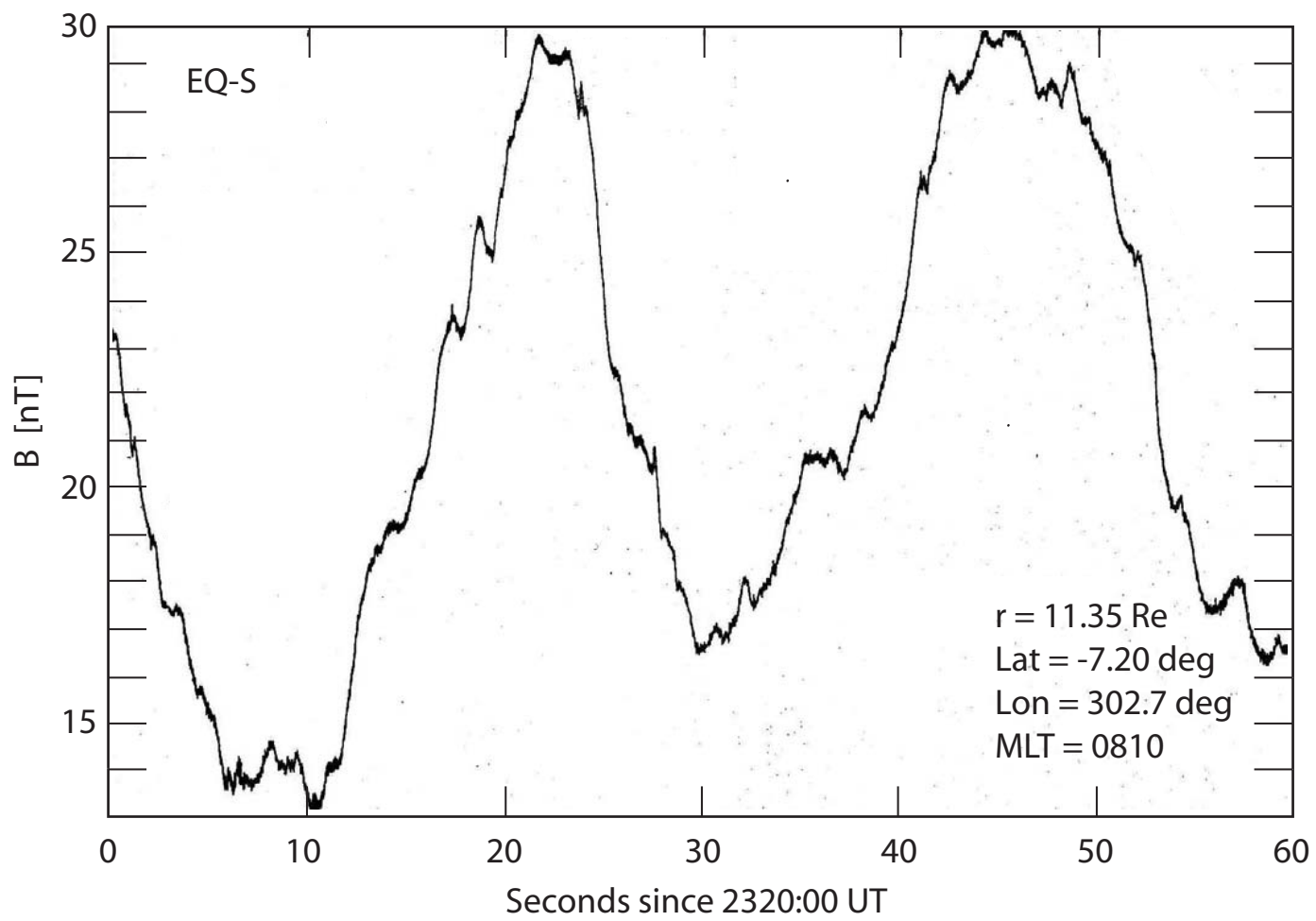

Fig. 5. A sequence of high-resolution magnetic field spacecraft observations of magnetosheath mirror modes close to the magnetopause recorded by Equator-S. The magnetic field exhibits large depressions the order of $66 \%$ in amplitude. In addition the shape of the magnetic field suggests that on the small scale the mirror structure is still in evolution showing deep erosions at its boundaries suggesting that at this stage magnetic flux is still expelled from the structure.

been identified. The critical magnetic field was found to scale as $B_{c}(\vartheta) \sim|\vartheta|^{\frac{1}{2}}$, and the penetration depth of the magnetic field into the bubbles scales as $|\vartheta|^{-\frac{1}{4}}$ both slightly different from scaling in metallic superconductors. These dependencies suggest that the low-temperature behavior in superconductors in the high-temperature plasma case corresponds to a low-temperature behavior in terms of $\vartheta$.

An interesting extension of these considerations is offered by application to collisionless reconnection which frequently is believed to be caused by generation of localized anomalous resistivity driven by small-scale current instabilities (Sagdeev, 1979a; Treumann, 2001). Measurements have so far not provided any convincing clue for anomalous resistivity (LaBelle and Treumann, 1988; Treumann et al., 1991; Bale et al., 2002). They support purely collisionless Halldominated reconnection (Fujimoto et al., 1997; Nagai et al., 2001; Øieroset et al., 2001; Runov et al., 2003). Also, numerical simulations (Hesse et al., 1999; Shay et al., 1999; Drake et al., 2003; Scholer et al., 2003; Jaroschek et al., 2004; Pritchett and Coroniti, 2004) do not support the generation of substantial anomalous resistivity.

The plasma involved in reconnection in space is manifestly collisionless and thus ideally conducting, and the problem of breaking the frozen-in state of the plasma is the same as that in the collisionless mirror mode. The main difference is that in the mirror mode the magnetic fields are parallel, and ini- tially no transverse current is flowing, while in reconnection the fields to both sides of the transverse current are (at least to some degree) antiparallel. In both cases the typical scales are identical, being the ion inertial scale (we note in passing that the ions on this scale become non-magnetized and Hall effects arise as the electrons are still tied to the magnetic field. This happens when $r_{c i}>\lambda_{i}$ which corresponds to the weak condition $\beta_{\perp}>1$ which is satisfied in mirror modes and in reconnection without or with only weak guide fields. Here ion inertia dominates over the ion-gyromagnetic effects causing the ions to decouple from the field and follow their own inertia. In particular, in the presence of electric fields on the ion inertial scale the ions will experience acceleration in the direction of the electric field).

Notably, Hall currents do not provide the diamagnetic effect required for annihilating the magnetic field in reconnection; the Hall magnetic field is transverse to the reconnection field. The situation is somehow similar to that in mirror modes with the main difference that the contacting fields are parallel in the mirror mode case. Therefore, one may expect that in similarity to mirror modes a diamagnetic effect may be generated in collisionless reconnection causing localized annihilation of the fields whenever the field drops below a certain still unknown threshold value (we note that arguments for the existence of such a threshold had already been given long ago by Sagdeev (1979b) based on consid- 
erations of electron dynamics in current layers which in the case investigated there were containing a week normal magnetic component (not a guide field!) thereby ab initio presupposing the presence of some weak reconnection). At the current stage, investigation of this mechanism is beyond the present approach however, even though it is of greatest interest from the point of view of understanding the disposition of free energy in collisionless reconnection. We may return to its discussion in future publications.

We conclude that the observation of mirror modes in the magnetosheath which are still slightly oblique and still contain a substantial component of trapped particles suggests that these structures, in spite of their large amplitudes and thus highly nonlinear states, are still nonlinearly evolving. This is most convincingly seen in very high-time resolution measurements of mirror mode structures by Equator-S (see Fig. 5). These observations show that the structures exhibit depressions on smaller scales along their walls which may be related to a stage when the flux tube is not yet stretched sufficiently and magnetic flux is still expelled from the flux tube. This is supported by the fact that the turbulence in the magnetosheath is restricted to a relatively narrow region in space which is bound by the dynamically evolving nonstationary bow shock and the as well dynamically evolving non-stationary magnetopause. The former reforms continuously while the latter undergoes time variable reconnection and large-scale instabilities like the Kelvin-Helmholtz instability. Moreover, the magnetosheath flow is non-stationary and the magnetosheath itself is highly inhomogeneous such that one cannot speak of an infinitely extended stationary turbulence or turbulence embedded into a box. The expectation of observing non-evolving mirror structures is therefore unrealistic. Mirror structures in the magnetosheath are mutually interacting and also interacting with the magnetosheath boundaries and thus are frozen into flow under nonlinear evolution. Their Cluster data-based recent identification as apparently distinct non-cascading turbulent modes (Sahraoui et al., 2003) with dispersion only marginally satisfying the linear dispersion relation provides another argument for them to still be in evolution not having reached their final stationary state.

Acknowledgements. The authors acknowledge illuminating discussions on the subject of this paper with S. Bale, W. Baumjohann, M. Balikhin, A. Balogh, M. Gedalin, K.-H. Glassmeier, T. Horbury, M. Kivelson, E. Lucek, G. Paschmann, A. Runov, R. Sagdeev, M. Scholer, S. Schwartz, T. Phan, and P. Yoon. RT thanks the ISSI, Bern, for support during a Senior Visiting Scientist period. CHJ also belongs to the IMPRES on Astrophysics, Garching. ODC also belongs to the IMPRES on Solar-Systems Sciences, Lindau. OAP acknowledges support by the PPARC through grant PPA/G/S/2002/00094, by the Russian Fund for Basic Research through the Grant No. 02-05-64612, and a visiting scientist grant from MPE. RT also acknowledges the contributions of the late N. Sckopke at the early state of these investigations.

Edited by: A. Chian

Reviewed by: one referee

\section{References}

Bale, S. D., Mozer, F. S., and Phan, T.: Observation of lower hybrid drift instability in the diffusion region at a reconnecting magnetopause, Geophys. Res. Lett., 29, 33-1, 2002.

Baumgärtel, K.: Soliton approach to magnetic holes, J. Geophys. Res., 104, 28 295-28 308, 1999.

Baumgärtel, K., Sauer, K., and Dubinin, E.: Towards understanding magnetic holes: Hybrid simulations, Geophys. Res. Lett., 30, 4$1,2003$.

Baumjohann, W., Treumann, R. A., Georgescu, E., Haerendel, G., Fornacon, K.-H., and Auster, U.: Waveform and packet structure of lion roars, Annales Geophysicae, 17, 1528-1534, 1999.

Chandrasekhar, S. A., Kaufman, A. N., and Watson, K. M.: The stability of the pinch, Proc. Royal Soc. London, Series A, 245, 435-456, 1958.

Chisham, G., Burgess, D., Schwartz, S. J., and Dunlop, M. W.: Observations of electron distributions in magnetosheath mirror mode waves, J. Geophys. Res., 103, 26765-26774, 1998.

Czaykowska, A., Bauer, T. M., Treumann, R. A., and Baumjohann, W.: Mirror waves downstream of the quasi-perpendicular bow shock, J. Geophys. Res., 103, 4747-4752, 1998.

Drake, J. F., Swisdak, M., Cattell, C., Shay, M. A., Rogers, B. N., and Zeiler, A.: Formation of electron holes and particle energization during magnetic reconnection, Science, 299, 873-877, 2003.

Fränz, M., Horbury, T., Génot, V., Moullard, O., Rème, H., Dandouras, I., Fazakerley, A. N., Korth, A., and Frutos-Alfaro, F.: Solitary waves observed by Cluster in the solar wind, in Solar Wind Ten, AIP Conf. Proc. 679, pp. 562-565, 2003.

Fujimoto, M., Nakamura, M. S., Shinohara, I., Nagai, T., Mukai, T., Saito, Y., Yamamoto, T., and Kokubun, S.: Observations of earthward streaming electrons at the trailing boundary of a plasmoid, Geophys. Res. Lett., 24, 2893-2896, 1997.

Gary, S. P.: The mirror and ion cyclotron anisotropy instabilities, J. Geophys. Res., 97, 8519-8521, 1992.

Glassmeier, K.-H., Motschmann, U., Mazelle, C., Neubauer, F., Sauer, K., Fuselier, S., and Acuna, M.: Mirror modes and fast magnetoacoustic waves near the magnetic pileup boundary of comet P/Halley, J. Geophys. Res., 98, 20 955-20 964, 1993.

Hasegawa, A.: Drift mirror instability in the magnetosphere, Phys. Fluids, 12, 2642-2650, 1969.

Hesse, M., Schindler, K., Birn, J., and Kuznetsova, M.: The diffusion region in collisionless magnetic reconnection, Phys. Plasmas, 6, 1781-1795, 1999.

Hill, P., Paschmann, G., Treumann, R. A., Baumjohann, W., and Sckopke, N.: Plasma and magnetic field behavior across the magnetosheath near local noon, J. Geophys. Res., 100, 95759584, 1995.

Huang, K.: Statistical Mechanics, J. Wiley, New York, 1995.

Hubert, D., Perche, C., Harvey, C. C., Lacombe, C., and Russell, C. T.: Observation of mirror waves downstream of a quasiperpendicular shock, Geophys. Res. Lett., 16, 159-162, 1989.

Huddleston, D. E., Strangeway, R. J., Blanco-Cano, X., Russell, C. T., Kivelson, M. G., and Khurana, K. K.: Mirror-mode structures at the Galileo-Io flyby: Instability criterion and dispersion analysis, J. Geophys. Res., 104, 17 479-17 490, 1999.

Jaroschek, C. H., Treumann, R., Lesch, H., and Scholer, M.: Fast reconnection in electron positron pair plasmas, Phys. Plasmas, 11, 1-13, doi: 10.1063/1.1644 814, 2004.

Kaufman, R. L., Horng, J. T., and Wolfe, A.: Large-amplitude hydromagetic waves in the inner magnetosheath, J. Geophys. Res. 
75, 4666-4684, 1970.

LaBelle, J. and Treumann, R. A.: Plasma waves at the dayside magnetopause, Space Sci. Rev., 47, 175-202, 1988.

Lacombe, C. F., Pantellini, G. E., Hubert, D., Harvey, C., Mangeney, A., Belmont, G., and Russell, C. T.: Mirror and Alfvénic waves observed by ISEE 1-2 during crossings of the Earth's bow shock, Annales Geophysicae, 10, 772-784, 1992.

Lifschitz, E. M. and Pitaevskii, L. P.: Statistical Physics, Part 2, vol. 9 of L. D. Landau and E. M. Lifschitz, Course of Theoretical Physics, Butterworth-Heinemann, Elsevier, Oxford, UK, 1998.

Lucek, E., Dunlop, M., Horbury, T., Balogh, A., Brown, P., Cargill, P., Carr, C., Fornacon, K. H., Georgescu, E., and Oddy, T.: Cluster magnetic field observations in the magnetosheath: four-point measurements of mirror structures, Annales Geophysicae, 19, 1421, 2001.

Lucek, E. A., Dunlop, W. M., Balogh, A., Cargill, P., Baumjohann, W., Georgescu, E., Haerendel, G., and Fornacon, K.-H.: Mirror mode structures observed in the dawnside magnetosheath by Equator-S, Geophys. Res. Lett., 26, 2159, 1999.

Lühr, H. and Klöcker, N.: AMPTE IRM observations of magnetic cavities near the magnetopause, Geophys. Res. Lett., 14, 186189,1987

Nagai, T., Shinohara, I., Fujimoto, M., Hoshino, M., Saito, Y., Machida, S., and Mukai, T.: Geotail observations of the Hall current system: Evidence of magnetic reconnection in the magnetotail, J. Geophys. Res., 106, 25 929-25 950, 2001.

Øieroset, M., Phan, T. D., Fujimoto, M., Lin, R. P., and Lepping, R. P.: In situ detection of collisionless reconnection in the Earth's magnetotail, Nature, 412, 414-417, 2001.

Pokhotelov, O. A., Treumann, R. A., Sagdeev, R. Z., Balikhin, M. A., Onishchenko, O. G., Pavlenko, V. P., and Sandberg, I.: Linear theory of the mirror instability in non-Maxwellian space plasmas, J. Geophys. Res., 107, doi:10.1029/2001-JA-009 125, 2002.

Pokhotelov, O. A., Sandberg, I., Sagdeev, R. Z., Treumann, R. A., Onishchenko, O. G., Balikhin, M. A., and Pavlenko, V. P.: Slow drift mirror modes in finite electron-temperature plasma: Hydrodynamic and kinetic drift mirror instabilities, J. Geophys. Res., 108, doi:10.1029/2002JA009 651, 2003.

Pokhotelov, O. A., Sagdeev, R. Z., Balikhin, M. A., and Treumann, R. A.: The mirror instability at finite ionLarmor radius wavelengths, J. Geophys. Res., 109, A09213, doi:10.1029/2004JA010 568, 2004.

Pritchett, P. L. and Coroniti, F. V.: Three-dimensional collisionless magnetic reconnection in the presence of a guide field, J. Geophys. Res., 109, A01 220, doi:10.1029/2003JA009 999, 2004.

Rudakov, L. I. and Sagdeev, R. Z.: A quasi-hydrodynamic description of a rarefied plasma in a magnetic field, in Plasma Physics and the Problem of Controlled Thermonuclear Reactions, vol. 3, p. 321, Pergamon Press, Tarrytown, N.Y., 1958.

Runov, A., Nakamura, R., Baumjohann, W., Treumann, R. A., Zhang, T. L., Volwerk, M., Vörös, Z., Balogh, A., Glassmeier, K.-H., Klecker, B., Rème, H., and Kistler, L.: Current sheet structure near magnetic X-line observed by Cluster, Geophys. Res. Lett., 30, 1579, doi:10.1029/2002GL016 730, 2003.

Russell, C. T., Riedler, W., Schwingenschuh, K., and Yeroshenko, Y.: Mirror instability in the magnetosheath of comet Halley, Geophys. Res. Lett., 14, 644-648, 1987.
Russell, C. T., Huddleston, D. E., Strangeway, R. J., Blanco-Cano, X., Kivelson, M. G., Khurana, K. K., Frank, L. A., Paterson, W., Gurnett, D. A., and Kurth, W. S.: Mirror-mode structures at the Galileo-Io flyby: Observations, J. Geophys. Res., 104, $17471-$ $17478,1999$.

Sagdeev, R. Z.: The 1976 Oppenheimer lectures: Critical problems in plasma astrophysics. I. Turbulence and nonlinear waves, Rev. Mod. Phys., 51, 1-9, 1979a.

Sagdeev, R. Z.: The 1976 Oppenheimer lectures: Critical problems in plasma astrophysics. II. Singular layers and reconnection, Rev. Mod. Phys., 51, 11-20, 1979b.

Sahraoui, F., Pincon, J.-L., Belmont, G., L. Rezeau, N. C.-W., Robert, P., Mellul, L., Bosqued, J. M., Balogh, A., Canu, P., and Chanteur, G.: ULF wave identification in the magnetosheath: The k-filtering technique applied to Cluster II data, J. Geophys. Res., 108, doi:10.1029/2002JA009 587, 2003.

Scholer, M., Sidorenko, I., Jaroschek, C., Treumann, R. A., and Zeiler, A.: Onset of magnetic reconnection in thin current sheets: Three-dimensional particle simulations, Phys. Plasmas, 10, 3521-3527, 2003.

Shay, M. A., Drake, J. F., Rogers, B. N., and Denton, R. E.: The scaling of collisionless, magnetic reconnection for large systems, Geophys. Res. Lett., 26, 2163-2166, 1999.

Southwood, D. J. and Kivelson, M. G.: Mirror instability: 1. Physical Mechanism of linear instability, J. Geophys. Res., 98, 91819187, 1993.

Tajiri, M.: Propagation of hydromagnetic waves in collisionless plasma, J. Phys. Soc. Japan, 2, 1482-1497, 1967.

Treumann, R. A.: Origin of resistivity in reconnection, Earth Planets Space (Japan), 53, 453-462, 2001.

Treumann, R. A. and Baumjohann, W.: Advanced Space Plasma Physics, Imperial College Press, London, 1997.

Treumann, R. A., Brostrom, L., LaBelle, J., and Sckopke, N.: The plasma wave signature of a "magnetic hole" in the vicinity of the magnetopause, J. Geophys. Res., 95, 19 099-19 114, 1990.

Treumann, R. A., LaBelle, J., and Pottelette, R.: Plasma diffusion at the magnetopause, the case of lower hybrid drift waves, J. Geophys. Res., 96, 16009-16013, 1991.

Tsurutani, B. T., Smith, E. J., Anderson, R. R., Ogilvie, K. W., Scudder, J. D., Baker, D. N., and Bame, S. J.: Lion roars and nonoscillatory drift mirror waves in the magnetosheath, J. Geophys. Res., 87, 6060-6072, 1982.

Tsurutani, B. T., Lakhina, G. S., Smith, E. J., Buti, B., Moses, S. L., Coroniti, F. V., Brinca, A. L., Slavin, J. A., and Zwickl, R. D.: Mirror mode structures and ELF plasma waves in the GiacobiniZinner magnetosheath, Nonlin. Proc. Geophys., 6, 229-234, 1999.

Winterhalter, D., Neugebauer, M., Goldstein, B., Smith, E., Bame, S., and Balogh, A.: Ulysses field and plasma observations and magnetic holes in the solar wind and their relation to mirrormode structures, J. Geophys. Res., 99, 23 371-23 381, 1994.

Yoon, P. H.: Quasi-linear evolution of Alfvén-ion-cyclotron and mirror instabilities driven by ion temperature anisotropy, Phys. Fluids, B4, 3627-3637, 1992. 